\section{A New 'Deleted' Rh-Chromosome}

Is 1950, Race, Sanger and Selwyn reported ${ }^{1}$ a very unusual $R h$ phenotype characterized by an extra. ordinary strong reaction with anti-D sera and completely negative reaction with anti-C, anti-c, anti-E and anti-e sera. The case was explained as the homozygous form of a partially deleted $R h$ chromosome, designated $D--$. Since then several examples of this chromosome have been reported, and further other partial deletions have been found ${ }^{2}$.

Recently an apparent mother-child exclusion was found in our laboratory. In a legal paternity case the mother was of phenotype $D+C-c+E+e-$ the child of phenotype $D+C+c-E-e+$. The results were checked with a number of different anti-Rh sera both in saline and using papainized cells and the indirect Coombs reaction. The possibility of an error while taking the blood sample was excluded by investigating now samples from the people concerned. The child was born in hospital, but the risk of an interchange of babies in the maternity ward was negligible, as the child was the only premature baby in the ward at that time. However, the ensuing investigation of the family definitely established the kinship of mother and child. The $R h$ types of the family, which were rather surprising, are shown in the following pedigree, + signifying positive and - signifying negative reaction with the corresponding antibodies.
A detailed report on the case and the results will be published later. I wish to thank Dr. A. E. Mourant and Dr. D. Parkin, of the Blood Group Reference Laboratory, Lister Institute, London, who kindly checked the results.

Serological Department,

K. Henningsen

University Institute of

Forensic Medicine,

Copenhagen. Oct. 14.

1 Race, R. R., Sanger, R., and Selwyn, J. G., Nature, 168, 520 (1950). ' Wiener, A. S., Gordon, E. B., and Cohen, I., Amer. J. Hum. Genet., B. T., and Hill, G. H., Amer. J. Clin. Path., 24. 202 (1954). Gunson, $\mathrm{H}$. H, and Donohue W. W. Vox Sang. (in the press). Henningsen, $\mathbf{K}$., Vox Sang. (in the press).

\section{Mitosis and Chromosome Numbers in the Euglenineae (Flagellata)}

NUClear division and the associated division of the cell and cell organelles have been examined in thirty-two species of the Euglenineae, drawn from thirteen genera. The main findings on the structure and mechanics of mitosis in this group are as follows.

Nuclear division in the Euglenineas is a true mitosis in that :

(1) Individualized chromosomes duplicate themselves longitudinally.

(2) Sister chromatids segregate to opposite poles.

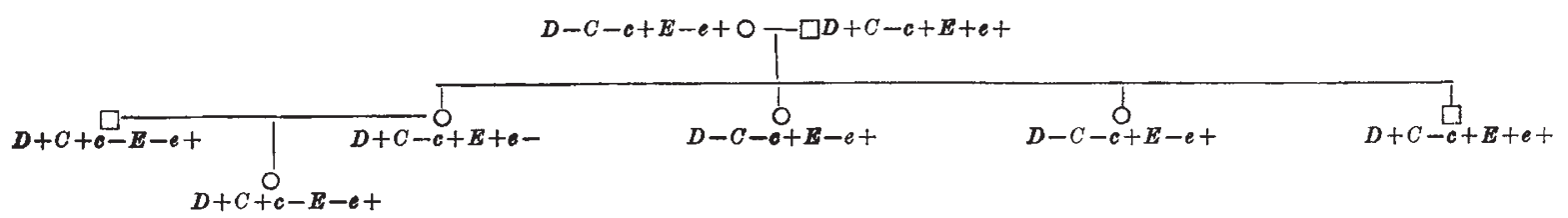

It is seen that there is an apparent exclusion of maternity in the first generation as well as in the second, the $R$ h-negative grandmother having a daughter who appears to be of genotype $R_{2} R_{2}$ or $R_{2} R^{\prime \prime}$. We knew that the irregularity affected the $C c$ as well as the Ee loci, but the really unexpected finding was, of course, that the grandmother was $D$-negative. The most simple explanation is a new, partially deleted chromosome involving $d(d--)$, or a triple deletion ( - - - ). It is of course impossible to ascertain if $d$ is present or not, as long as anti- $d$ is not available. It is further obvious that one of the criteria on the hitherto known partial $R h$-deletions, the enhanced agglutinability of the $D$-receptor, cannot be put to use in the diagnosis of $d$ - - or - - -. However, it is to be expected that the erythrocytes from persons carrying the abnormal chromosome would show single-dose reactivity with regard to the $R h$ receptors, which according to the phenotype appear to be homozygous. Titration experiments against the pertinent sera appear to substantiate this expectation. According to these experiments the genotypes of the family are the following, - denoting chromosome $d-$ - or - - - :

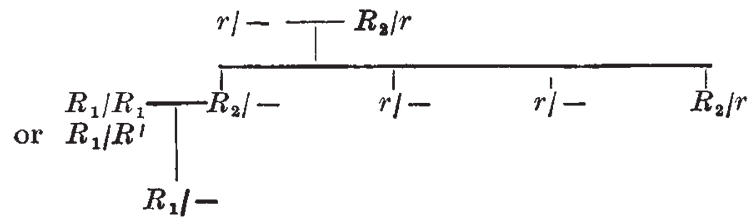

The mitosis differs from the 'classical' form in the following ways :

(1) An achromatic body in the nucleus, the 'endosome', persists throughout mitosis, dividing as a structure distinct from the chromosomes (Fig. 1).

(2) The endosome retains its ribonucleic acid during mitosis.

(3) There are no centromeres or spindle.

(4) Mitosis and cell activity are unaffected by 4 per cent colchicine, or by ionizing radiations at doses of up to $100,000 \mathrm{r}$.

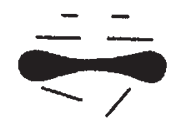

A

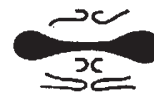

C

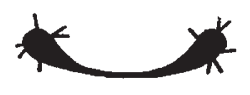

E

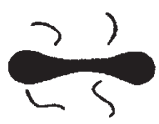

B

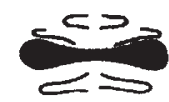

D

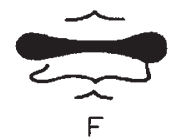

Fig. 1. Chromatid shapes during mitotic anaphsee in the Kuglenineae. The dividing endosome is also shown 